\title{
Alterações psicomotoras por meio das aulas de Educação Física em crianças de 8 a 9 anos de uma escola municipal de Anápolis-GO
}

\author{
Psychomotor changes through the physical education classes in \\ children of 8 to 9 years of a school hall of Anápolis-GO
}

Patrícia Espíndola Mota Venâncio', David Henrique de Souza Matias', Grassyara Pinho Tolentinho, Gabriel Dutra de Jesus Siqueira', Samuel Vidal', Iransé Oliveira Silva' 'Universidade Unievangélica Centro Universitário de Anápolis, GO, Brasil.

Recebido em: março 2015 / Aceito em: junho 2015

venanciopatricia@hotmail.com

\section{RESUMO}

Objetivo: comparar as alterações psicomotoras por meio das aulas de Educação Física, em crianças de 8 a 9 anos de uma escola municipal de Anápolis-Go. Método: a amostra foi composta por 31 alunos de uma escola municipal de Anápolis-GO. Incluiu a realização da bateria psicomotora de Oliveira, que avaliou coordenação motora, equilíbrio, esquema corporal, lateralidade, estruturação espaço-temporal. Houve uma intervenção de 06 meses, e duas avaliações pré e pós. A amostra foi submetida a duas aulas de Educação Física por semana, com duração de 50 minutos, envolvendo atividades lúdico-recreativas, com base nas necessidades encontradas no primeiro teste, finalizando com a reavaliação psicomotora a fim de constatar as alterações ocorridas durante a intervenção. Foi realizado o test "t de Mann-Whitney para comparar o pré e pós avaliação por meio do software SPSS 21.0, com nível de significância de $p \leq 0,05$. Resultados: constatou-se que as crianças analisadas se encontravam dentro da faixa de aprendizagem. Melhoras significativas na coordenação motora e equilíbrio, no esquema corporal e na estrutura espacial foram observadas a partir da intervenção psicomotora de seis meses. Considerações finais: os aspectos psicomotores dos escolares, após o desenvolvimento de práticas corporais que envolviam elementos psicomotores por meio das aulas de Educação Física, aprimoraram os parâmetros esperados para a sua faixa etária.

Palavras-chave: Psicomotricidade; Escolares; Educação Física.

\section{ABSTRACT}

Objective: compare psychomotor changes through physical education classes in 8-9 years children from a municipal school in Anápolis (Goiás). Method: sample was composed by 31 students of a municipal school. Oliveira's Psychomotor Battery was performed evaluating motor coordination elements, balance, body scheme, laterality and space-time structure. There was an intervention of 6 months, and two pre and post assessments. Students took part in two physical education classes per week lasting 50 minutes and involving ludic and recreational activities, based on the needs found in the pre-assessment. In the end a new psychomotor assessment was performed in order to determine changes during the intervention. Mann-Whitney test was conducted for comparing pre and post moment using SPSS 21.0 software with $p \leq 0.05$. Results: children analyzed were within the range of learning and psychomotor intervention. Significant improvements in all components were observed after six months of psychomotor classes. Closing remarks: after development of body practices involving psychomotor elements through physical education classes children's psychomotor aspects get improved, considering parameters expected for their age group.

Keywords: Psychomotor; School; Physical Education. 


\section{INTRODUÇÃO}

A psicomotricidade é toda expressão corporal e intelectual da criança em relação ao mundo. Ela veio para contribuir junto à interpretação das ações corporais, tais como: diálogo, gestos, movimento e afetividade, para que, por meio das ações observáveis, seja possível entender como a criança manifesta, expressa e aperfeiçoa seus conhecimentos. A psicomotricidade não pretende ser exclusiva e nem solucionar todos os problemas, mas sim, ser uma auxiliadora/método capaz de somar à evolução cognitiva, intelectual, expressiva e motora da criança. ${ }^{2}$

A psicomotricidade está em todas as atividades que envolvem a motricidade humana, pois dificilmente pode-se separar a criança do movimento. Este movimento habitual está ligado ao desenvolvimento corporal. O movimento é a base fundamental para o desenvolvimento da aprendizagem. Os elementos psicomotores: esquema corporal, estruturação espacial, lateralidade, orientação temporal, coordenação motora grossa e fina são aspectos que interferem diretamente no aprendizado da escrita e leitura, pois quando pouco desenvolvidos, ocasionam alguns déficits na compreensão de conhecimentos básicos, uma vez que haverá interferência na associação das noções de tempo, espaço, conceitos e ideias; enfim, na aquisição de conhecimentos, que no futuro influenciará na criação de barreiras contra os processos de aprendizado. ${ }^{3}$

$\mathrm{O}$ estudo psicomotor tende a beneficiar as crianças na escola devido ao amplo leque de recursos capazes de influenciar o desenvolvimento das funções neuropsicológicas, impactando diretamente no processo de maturação e proporcionando meios que as capacitem para aquisição de habilidades e conhecimentos, tanto motores, quanto cognitivos e afetivos, respeitando totalmente os potenciais intelectuais, sociais, motores e psicomotores. Devidamente inserida na ação pedagógica, a psicomotricidade abrange todos os alunos, principalmente os alunos considerados com déficits de atenção, "alunos-problema", "lentos", "preguiçosos" e entre outras adjetivações utilizadas como rótulos, sem uma análise minuciosa e criteriosa. ${ }^{4}$

A proposta da Educação Física com abordagem psicomotora vem com o objetivo de relacionar o movimento ao fator cognitivo, sendo que a Educação Física é a área que envolve a saúde nas suas totalidades, tais como, aperfeiçoamento, manutenção e reabilitação, por meio de atividades básicas como andar, correr, saltar, entre outros atos da vida diária, que por sua vez buscam o desenvolvimento motor. Partindo dessa premissa, a origem da psicomotricidade, tem como função abordar na Educação Física, uma visão ampla e mudar o conceito de que o movimento é algo realizado de forma individualizada. A Psicomotricidade busca entender o que está por de traz do movimento, tais como: a comunicação e a expressão corporal. A proposta de trabalhar a Psicomotricidade de forma multidisciplinar destina-se a auxiliar a criança a tomar consciência de seu corpo, da lateralidade, localização no espaço, dominar seu tempo e adquirir, habilmente, a coordenação de seus gestos e movimentos. Tais alvos fazem com que a Educação Física e a Psicomotricidade sejam elementos importantes para desenvolvimento psíquico e motor de um indivíduo. ${ }^{5}$
Neste sentido, o estudo se justifica ao pesquisar a importância da abordagem para o desenvolvimento psicomotor das crianças, envolvendo fatores responsáveis e indispensáveis para maturação, não podendo passar despercebido na nossa atuação profissional como metodologia inclusiva nos conteúdos da Educação Física. Nesta perspectiva, considerando os estudos sobre a temática na realidade nacional, o presente estudo busca comparar as alterações psicomotoras por meio das aulas de Educação Física, em crianças de 8 a 9 anos de uma Escola Municipal de Anápolis-GO.

\section{MÉTODO}

O presente estudo é uma pesquisa descritiva experimental. A população do estudo foi composta por 250 crianças de 08 a 09 anos de idade, totalizando uma pré-amostra de 31 alunos, 13 meninas e 18 meninos, estudantes de uma escola da rede pública de ensino da cidade de Anápolis-GO. Houve uma perda amostral de 15 alunos por motivos diversos, como: evasão escolar e por não estarem no período integral na escola. Foi utilizada a Bateria Psicomotora de Oliveira ${ }^{1}$ e aplicados os testes de coordenação global e fina, equilíbrio, esquema corporal, lateralidade, estruturação espacial, estruturação temporal, com intuito de identificar o nível psicomotor das crianças.

Os pais dos participantes ou responsáveis foram informados acerca dos procedimentos a serem adotados durante a pesquisa, bem como dos riscos e benefícios que poderiam resultar de sua participação. A cada responsável foi entregue um Termo de Consentimento Livre e Esclarecido (TCLE), contendo as informações mencionadas acima, conforme a Resolução 196/96 do Conselho Nacional de Saúde, sobre pesquisas com seres humanos, e a Declaração de Helsinque, de 1975.

A primeira fase do estudo consistiu em convidar a escola para participar da pesquisa. Após o convite, foi solicitado à diretora que escolhesse uma sala de aula, na qual os alunos aparentemente apresentassem maiores dificuldades de aprendizagem ou idade correspondente à pesquisa. Posterior à escolha, foi feito o convite às crianças a participarem do estudo, com o encaminhamento do TCLE, a todos os envolvidos.

Após o retorno do TCLE, com as respectivas assinaturas, foi iniciada a pesquisa, primeiramente com a coleta dos dados, por meio da bateria psicomotora de Oliveira. ${ }^{1}$ As crianças foram avaliadas individualmente em uma sala reservada e arejada, em que não tinha interferência externa, evitando o desvio de atenção, que poderia influenciar na aplicação dos testes. Estes tiveram a duração de 30 a 40 minutos para cada criança. As avaliações foram realizadas pelos pesquisadores treinados, os quais eram responsáveis pela pesquisa. $\mathrm{O}$ segundo momento foi a identificação dos déficits apresentados pelas crianças, nos resultados dos testes psicomotores, seguido de intervenção no período de seis meses, com atividades lúdico-recreativas como: brinquedos cantados, circuitos psicomotores, brincadeiras antigas que pudessem aproximar da relação cultural pelas propostas de atividades que os pais faziam na época de criança, coletânea de jogos que envolviam todos os elementos psicomotores, dentre outras. O terceiro 
momento, após a intervenção, consistiu na reavaliação psicomotora dos sujeitos participantes, utilizando-se a mesma bateria de testes e a tabulação dos dados para comparação dos resultados.

No tratamento dos dados, foi feita uma análise descritiva na forma de porcentagem e aplicado o teste de Mann-Whitney, por meio de software SPSS 21.0, adotando o nível de significância de p 0,05.

\section{RESULTADOS}

A seguir, estão apresentados os resultados separadamente para cada componente psicomotor, destacando que não houve amostra suficiente para a comparação entre os sexos.

A Tabela 01 mostra os resultados quanto à classificação da coordenação e equilíbrio, observando-se diferença significativa antes e após a intervenção $(p<0,001)$. Constatou-se que a maioria dos avaliados, no primeiro momento, foram classificados como "reorganização do corpo percebido", correspondendo a coordenação motora e equilíbrio de 8 a 9 anos, com 62,5\%. Após a intervenção de seis meses, a maior parte $(81,2 \%)$ ficou classificada como "indícios de presença de corpo representado" (10 a 11 anos).

$\mathrm{Na}$ Tabela 02 , observam-se os resultados quanto à classificação do esquema corporal. Constatou-se que a maioria dos avaliados $(56,3 \%)$, no primeiro momento, foram classificados como "reorganização do corpo percebido". Após a intervenção, houve diminuição de escolares nesta categoria e aumento significativo $(p=0,013)$ do percentual na classificação “indícios de presença de corpo representado" $(49,9 \%)$.

Com relação à lateralidade (Tabela 3), não foram observadas diferenças significativas após a intervenção, sendo que os escolares foram classificados, tanto no pré-teste $(50,0 \%)$, quanto no pós-teste $(50,0 \%)$, como "reorganização do corpo percebido". Porém, ressalta-se que houve diminuição nas categorias que evidenciam lateralidade em idades inferiores ( 5 a 6 anos e 7 anos), bem como um aumento nas classes que classificam a lateralidade em idades superiores (10 a 11 anos e a partir de 12 anos).

A Tabela 04 mostra os resultados quanto à classificação da estrutura espacial, em que foram observadas diferenças significativas após a intervenção $(p=0,023)$. Observou-se que, no momento pré-teste, a maioria dos escolares estavam classificados como "indícios de presença de imagem de corpo percebido" (5 a 6 anos), com $31,3 \%$. Após a intervenção, houve um predomínio de escolares na classe "indícios de presença de corpo representado" (10 a 11 anos), com 62,5\%.

Tabela 1 - Classificação do Elemento Coordenação Motora e Equilíbrio.

\begin{tabular}{lcc}
\hline Coordenação motora e equilíbrio & Pré (\%) & Pós (\%) \\
\hline Imagem de corpo vivido (até 3 anos) & -- & - \\
Reorganização do corpo vivido (3 a 4 anos) & - & - \\
Indícios de presença de imagem de corpo percebido (5 a 6 anos) & 25,0 & -- \\
Imagem de corpo percebido (7 anos) & -- & -- \\
Reorganização do corpo percebido (8 a 9 anos) & 62,5 & 12,5 \\
Indícios de presença de corpo representado (10 a 11 anos) & 12,5 & $81,2 *$ \\
Imagem de corpo representado (a partir de 12 anos) & -- & 6,3 \\
Total & 100,0 & 100,0 \\
\hline${ }^{*} p<0,001$ & &
\end{tabular}

Tabela 2 - Classificação do Elemento Esquema Corporal.

\begin{tabular}{lcc}
\hline Esquema corporal & Pré (\%) & Pós (\%) \\
\hline Imagem de corpo vivido (até 3 anos) & -- & - \\
Reorganização do corpo vivido (3 a 4 anos) & -- & -- \\
Indícios de presença de imagem de corpo percebido (5 a 6 anos) & 25,0 & 6,3 \\
Imagem de corpo percebido (7 anos) & 6,3 & 6,3 \\
Reorganização do corpo percebido (8 a 9 anos) & 56,3 & 37,5 \\
Indícios de presença de corpo representado (10 a 11 anos) & 12,5 & $49,9 *$ \\
Imagem de corpo representado (a partir de 12 anos) & -- & -- \\
Total & 100,0 & 100,0 \\
\hline
\end{tabular}

Tabela 3 - Classificação do Elemento Lateralidade.

\begin{tabular}{lcc}
\hline Lateralidade & Pré (\%) & Pós (\%) \\
\hline Imagem de corpo vivido (até 3 anos) & - & - \\
Reorganização do corpo vivido (3 a 4 anos) & -- & - \\
Indícios de presença de imagem de corpo percebido (5 a 6 anos) & 6,3 & -- \\
Imagem de corpo percebido (7 anos) & 12,5 & -- \\
Reorganização do corpo percebido (8 a 9 anos) & 50,0 & 50,0 \\
Indícios de presença de corpo representado (10 a 11 anos) & 31,3 & 43,8 \\
Imagem de corpo representado (a partir de 12 anos) & -- & 6,3 \\
Total & 100,0 & 100,0 \\
\hline
\end{tabular}


Tabela 4 - Classificação do Elemento Estrutura Espacial.

\begin{tabular}{|c|c|c|}
\hline Estruturação espacial & Pré (\%) & Pós (\%) \\
\hline Imagem de corpo vivido (até 3 anos) & -- & -- \\
\hline Reorganização do corpo vivido (3 a 4 anos) & 6,3 & -- \\
\hline Indícios de presença de imagem de corpo percebido (5 a 6 anos) & 31,3 & 6,3 \\
\hline Imagem de corpo percebido (7 anos) & 12,5 & -- \\
\hline Reorganização do corpo percebido (8 a 9 anos) & 25,0 & -- \\
\hline Indícios de presença de corpo representado (10 a 11 anos) & 25,0 & $62,5^{*}$ \\
\hline Imagem de corpo representado (a partir de 12 anos) & -- & 31,3 \\
\hline Total & 100,0 & 100,0 \\
\hline
\end{tabular}

Tabela 5 - Classificação do Elemento Estrutura Temporal.

\begin{tabular}{lcc}
\hline Estruturação temporal & Pré (\%) & Pós (\%) \\
\hline Imagem de corpo vivido (até 3 anos) & -- \\
Reorganização do corpo vivido (3 a 4 anos) & -- & 12,5 \\
Indícios de presença de imagem de corpo percebido (5 a 6 anos) & -- \\
Imagem de corpo percebido (7 anos) & 50,0 \\
Reorganização do corpo percebido (8 a 9 anos) & 31,3 \\
Indícios de presença de corpo representado (10 a 11 anos) & 6,3 \\
Imagem de corpo representado (a partir de 12 anos) & 31,3 \\
Total & 12,5 & 100,0 \\
\hline
\end{tabular}

$\mathrm{Na}$ avaliação da estrutura temporal (Tabela 5), não foram observadas diferenças significativas, sendo que a maioria dos escolares avaliados como "reorganização do corpo percebido" (8 a 9 anos), no pré-teste, mantiveram esta classificação após a intervenção.

\section{DISCUSSÃO}

Observou-se, no presente estudo, que a intervenção com atividades lúdico-recreativas, durante 6 meses, demonstrou melhora na coordenação motora e equilíbrio, no esquema corporal e na estrutura espacial. Ressalta-se também que não houve déficits relevantes nos elementos psicomotores, os quais caracterizaram os alunos estudados dentro da normalidade para sua faixa etária, tanto no primeiro momento, quanto no segundo, destacando que as crianças do presente estudo estão com o desenvolvimento psicomotor adequado para sua faixa etária.

Já no estudo de Almeida, ${ }^{6}$ o qual teve como objetivo verificar o perfil psicomotor de 28 crianças com idade entre 7 a 9 anos, estudantes do projeto Piá Ambiental, no município de Campo Magro-PR, constatou, por meio da bateria psicomotora de Fonseca, ${ }^{7}$ que os alunos demonstraram dificuldades em esquema corporal, estruturação espaço-temporal e coordenação global. Venâncio et al., ${ }^{8}$ em estudo envolvendo 27 crianças na idade entre 6 e 8 anos de Anápolis-GO, objetivando avaliar o efeito das aulas de psicomotricidade no perfil psicomotor em escolares, demonstraram que a maioria dos escolares foram classificados como muito inferior no quesito motricidade fina; normal médio em motricidade global e equilíbrio; inferior em esquema corporal e normal baixo em organização espacial e organização temporal.

O presente estudo demonstrou melhora nos componentes psicomotores como resultado da intervenção de um professor de Educação Física, ressaltando a relevância e o diferencial da atuação compromissada de um professor de Educação Física. Ferrari e Moretti, ${ }^{9}$ em estudo realizado em Mirante do Paranapanema-SP, que teve como objetivo analisar o trabalho do profissional de Educação Física na formação integral dos alunos da primeira série do Ensino Fundamental, bem como verificar se o desenvolvimento dos aspectos afetivo, cognitivo e motor estão sendo trabalhados na referida disciplina, demonstraram que a disciplina de Educação Física, na forma metodológica, não contemplava as dimensões essenciais para o desenvolvimento da criança devido à formação tecnicista recebida pelo professor de Educação Física. O estudo demonstrou um perfil psicomotor abaixo do esperado, significando que poderão surgir dificuldades de aprendizagem na vida escolar dessas crianças. De forma semelhante, estudo realizado em Samambaia-DF também demonstrou que os alunos dos anos iniciais do Ensino Fundamental apresentaram desenvolvimento psicomotor abaixo do padrão esperado para idade cronológica. ${ }^{10}$

Por outro lado, estudo de Gonçalves, ${ }^{11}$ com o objetivo de caracterizar o perfil psicomotor de alunos do Programa Escolinhas Integrado - PEI/UNISINOS, de São Leopoldo-RS, destaca que crianças da terceira e quarta séries do Ensino Fundamental, avaliadas pelo instrumento de Fonseca, ${ }^{7}$ estavam classificadas com predomínio de perfil hiperpráxico (excelente) nos fatores psicomotores tonicidade, lateralidade e praxia fina, equilíbrio de perfil hiperpráxico e eupráxico (bom) no fator psicomotor praxia global. Resultados satisfatórios também foram encontrados por Barcelos e Almeida, ${ }^{12}$ os quais objetivaram delinear o perfil psicomotor em crianças e adolescentes de João Monlevade-MG, a fim de nortear o programa a ser elaborado para os grupos de três faixas etárias (G1- de 8 a 10 anos, G2 - de 11 a 13 anos e G3 - de 14 e 15 anos), em especial aqueles que demonstraram alterações relevantes nos elementos psicomotores. Os autores relataram uma predominância de valores dentro da faixa de normalidade para os 
alunos dentre os sete fatores psicomotores avaliados. Sanches et al. ${ }^{13}$ também verificaram, em crianças com 6 a 8 anos do interior de São Paulo, que todas se enquadravam no perfil psicomotor bom e normal. Por fim, Rosa Neto et al. ${ }^{14}$ também verificaram que $96 \%$ dos escolares estudados, no município de Florianópolis-SC, estavam dentro do esperado para a idade.

Já, Ferreira ${ }^{15}$ demonstrou a importância de analisar a avaliação psicomotora como instrumento de adaptação das crianças com o meio em que vivem. Os dados coletados, no município de Fortaleza-CE, apontam que das 6 crianças avaliadas, 4 atingiram o perfil psicomotor normal e as outras 2 crianças obtiveram o perfil psicomotor bom, seguindo a escala de pontos, ou seja, crianças sem grandes dificuldades psicomotoras que possam influenciar no comportamento.

\section{CONSIDERAÇÕES FINAIS}

De modo geral, foi concluído que o desenvolvimento psicomotor dos escolares encontra-se dentro dos parâmetros de normalidade esperados para a faixa etária. Quanto às alterações psicomotoras, após a intervenção de seis meses, as crianças apresentaram uma melhora significativa na coordenação motora e equilíbrio, no esquema corporal e na estrutura espacial.

Sugere-se que sejam realizadas novas pesquisas que possam analisar outros fatores que possam influenciar o desenvolvimento destas crianças nos testes psicomotores, os quais exigem percepção, raciocínio, interpretação e lógica, para avaliação mais apurada no sentido de compreender, além das aulas de Educação Física, outros fatores intrínsecos ou extrínsecos ao âmbito escolar.

\section{REFERÊNCIAS}

1. Oliveira $\mathrm{G}$ de $\mathrm{C}$. Avaliação psicomotora à luz da psicologia $\mathrm{e}$ da psicopedagogia. 10 ed. Petrópolis-RJ: Vozes. 2012.

2. Alves F. Como aplicar a Psicomotricidade: uma atividade multidisciplinar com amor e união. 4 ed. Rio de Janeiro: Wark. 2011.

3. Rossi FS. Considerações sobre a psicomotricidade na educação infantil. Rev Vozes dos Val da UFVJM Publicações
Acadêmicas-MG-Brasil. 2012

4. Oliveira $\mathrm{G}$ de $\mathrm{C}$. Psicomotricidade: educação e reeducação num enfoque psicopedagógico. 17 ed. Petrópolis-RJ: Vozes. Vozes; 2012.

5. Oliveira CS de, Barros CL de, Silva PM do N. Educação Física Escolar e a intervenção da psicomotricidade no processo de aprendizagem. 2013.

6. Almeida C. Perfil psicomotor de alunos com idade entre 7 a 9 anos. Congresso Nacional de Educação. 2009. p. 7277-87.

7. Venâncio PEM, Teixeira Junior J, Fernandes RM, Silva IO, Lima WA, Santos CGO, et al. Psicomotricidade e a Influência no Desenvolvimento de Crianças. Rev Científica Jopef - Ed Korppus - Curitiba/PR 2011; 11(1): 21-8.

8. Ferrari EC dos S, Moretti LHT. A Educação Física e a Educação Psicomotora na formação integral do aluno. IX Congr Nac Educ - EDUCERE III Encontro Sul Bras Psicopedag 2009; 6795-807.

9. Santos $A G$ da $S$ dos. Educação física e psicomotricidade nos anos iniciais do ensino fundamental da Escola 317 de Samambaia. Trab Conclusão Curso II e no Curso Licenciatura em Educ Física do Programa Pró-Licenciatura da Univ Brasília - Pólo Brasília - DF. 2013.

10. Gonçalves EF. Perfil psicomotor: caracterização de alunos da 30 e 40 séries do ensino fundamental do Núcleo Cruz Vermelha - PEI, Programa Escolinhas Integradas. Rev Digit Buenos Aires. 2008; (120).

11. Barcelos THS, Almeida $M$ de $C R$ e. Diagnose do perfil psicomotor em crianças e adolescentes de 8 a 15 anos de um projeto social da cidade de João Monlevade-MG. Mov - Rev Digit Educ Física 2008; 3(2): 1-11.

12. Sanches SO, Guerra LA, Luft CB, Andrade A. Perfil Psicomotor associado a aprendizagem escolar. Rev Digit Buenos Aires 2004; 10(79).

13. Rosa Neto F, Santos APM dos, Xavier RFC, Amaro KN. A Importância da avaliação motora em escolares: análise da confiabilidade da Escala de Desenvolvimento Motor. Rev Bras Cineantropom Desempenho Hum. SciELO Brasil 2010; 12(6): 422-7. DOI: 10.5007/1980-0037.2010v12n6p422.

14. Ferreira HS. Testes psicomotores na educação infantil-bateria psicomotora (BPM): um estudo de caso em crianças de uma escola particular. 2001. 100 f. Monografia (Especialização em Psicomotricidade), Univ Estadual do Ceará, Fortaleza-CE. 2001. 